Meta

Journal des traducteurs

Translators' Journal

\title{
Index des mots et sujets traités dans le volume 15
}

Volume 15, numéro 4, décembre 1970

URI : https://id.erudit.org/iderudit/003215ar

DOI : https://doi.org/10.7202/003215ar

Aller au sommaire du numéro

\section{Éditeur(s)}

Les Presses de l'Université de Montréal

ISSN

0026-0452 (imprimé)

1492-1421 (numérique)

Découvrir la revue

Citer ce document

(1970). Index des mots et sujets traités dans le volume 15. Meta, 15(4), 248-256.

https://doi.org/10.7202/003215ar d'utilisation que vous pouvez consulter en ligne.

https://apropos.erudit.org/fr/usagers/politique-dutilisation/ 


\section{INDEX DES MOTS ET SUJETS TRAITES DANS LE VOLUME 15}

\section{A clapet, 3, p. 174}

A passage direct, 3, p. 175.

A robinet-vanne, 3, p. 174

Abcès urineux, 2, p. 105

Abdomen urgent, 2, p. 103.

"Abimer (s') la santé 》, 2, p. 99.

Abréviations, 1, p. 48.

Abréviations techniques, 1, p. 54

ABS, 1, p. 42 .

Absence (đe mémoire), 1, p. 38

Abstraction, 1, p. 28, 37 .

Abstrait, 1, p. $5 ; 4$, p. 203

Académie française, 1, p. 53.

Accélération lexicologique, 1, p. 53.

Accent verbal, 1, p. 27

"Accommodation ", 1, p. 36

Acroparesthésie nocturne, 2, p. 97.

A.C.T.H., 2, p. 108

Action corrective, 1, p. 61 .

Action normative, 1, p. 61 .

Activité tropinique, 2, p. 96

Actualiser, 4, p. 222.

Adaptation culturelle, 2, p. 89.

Adaptation du français aux réalités contemporaines, 1, p. 63

Addisonisme, 2, p. 107.

Administration, 1, p. 39.

Aéroglisseur, 1, p. 65.

Affaires (langue des), 1, p. 48

Affection larvaire, 2, p. 104

Af iection valvulaire, 2, p. 99

Age, 4, p. 236

Agent de maîtrise, 1, p. 40 .

Alcotest, 1, p. 65 .

Alimentation, 3 , p. 175.

Alimentation directe, 3 , p. 175.

Alimentation en retour, 3 , p. 175 .

Allantoïdien, 2, p. 105.

Allemand, 1, p. 55

Amblyopie crépusculaire, 2, p. 97.

Ame, 1, p. 6

Aménagement des ressources linguistiques du français, 1, p. 62

“Amocher quelqu'un », 2, p. 99.

Analyse, 1, p. 41 .

Analyse avantages-coûts, 4, p. 223

Analyse contrastive, 4, p. 203, 207.

Analyse coûts-bénéfices, 4, p. 223.

Analyse coûts-efficacité, 4, p. 223.

Analyse des programmes, 4, p. 222.

Analyse stylistique, 1, p. 47 .

Anamèse, 2, p. 101

Anarthrie, 2, p. 97.

Anastomose termino-latérale, 2, p. 98 .

Ancien Testament, 1, p. 3

Androgène, 2, p. 105 .

Anémie splénique infectieuse, 2, p. 105.

Angineux, 2, p. 104.

Anglicismes, 1, p. 63

Anglicismes sémantiques, 1, p. 36 .
Année préliminaire, 4, p. 231.

Anomalie, 2, p. 99.

Anti-B.K., 2, p. 108

Antigalactogène, 2, p. 105.

Antihistaminique, 2, p. 105.

Antiquité mésopotamienne, 1, p. 32.

Antivol, 4, p. 232

Antivol contact, 4, p. 232

Antivol contact démarreur, 4, p. 232

Aortique, 2, p. 104.

Aphasie motrice, 2, p. 97.

Apophyse, 2, p. 106.

Appareil urinaire, 2, p. 105.

Appendice, 2, p. 106.

Appendiculaire, 2, p. 104.

Apprentissage, 1, p. 40.

"Approche ", 2, p. 102

"Approcher quelqu'un", 1, p. 37.

AR, 4, p. 233.

Arabe, 3, p. 184

Archéologie, 1, p. 7

Architecture, 4, p. 240.

Argot, 4, p. 240

Armoires d'incendie, 3, p. 175.

Arsis, 1, p. 18

Artère mentonnière, 2, p. 104.

Articulation, 2, p. 97

Articulation orbiculaire, 2, p. 98.

Arts du spectacle, 4, p. 240.

"Assigner quelqu'un », 1, p. 39.

Assistanat, 1, p. 65

Assurances sur la vie, 1, p. 55

Astéatose cutanée, 2, p. 97.

Atlas économique, 1 , p. 55 .

Attaque (d'), 2, p. 102.

Atteindre, 2, p. 99.

Attribution des tâches, 1, p. 40.

Automobile, 4, p. 231.

Avatar, 1, p. 64 .

Avertisseur optique, 4, p. 232.

Avertisseur sonore, 4, p. 232

Avoir le goût de, 1, p. 38

Axillaire, 2, p. 104.

Baccalauréat, 3, p. 167.

Bain statique, 2, p. 107

Ballon, 2, p. 96.

Bandelette, 2, p. 106

Banquette, 1, p. 37.

Barrage de prise, 3, p. 175.

Barrière hémato-encéphalique, 2, p. 98 .

Basedowisme, 2, p. 107.

Bâtiment, 4, p. 240.

Bâtiment voyageurs, 1, p. 42.

B.C.G., 2, p. 108.

Beau, 3, p. 150.

Bible (traduction de la), 1, p. 3 .

Bible de Jérusalem, 1, p. 4.

Bible hébraïque, 1, p. 34

Bibliographie, 1 , p. $54 ; 2$, p. $118 ; 3$, p. 183 ; 4 , p. 239. 
Biennale de Liège, 1, p. 60.

Biliaire, 2, p. 104.

Bilieux, 2, p. 104.

B.K., 2, p. 108.

"Blanc $", 1$, p. 37.

"Blanc de mémoire », 1, p. 37.

Blastoderme, 2, p. 106.

Bloc de commande, 4, p. 233.

Boisson gazeuse, 1, p. 37.

Bon a.ppétit, 2, p. 93 .

Bordereau, 2, p. 112.

Bordure, 2, p. 106.

Borne-fontaine, 3, p. 175

Bouche d'incendie, 3, p. 175

Bouchon, 3, p. 174 .

Bouclé, 3, p. 174.

Bourgeon, 2, p. 106.

Bourrelet, 3, p. 174.

Bourrelet médulaire, 2, p. 106

"Bouteille de bébé », 2, p. 117.

Bouton de porte, 3, p. 182 .

Boyau, 3, p. 173.

Branchement d'eau, 3, p. 175.

"Breuvage ", 1, p. 37

Brevets (termes de), i, p. 52.

Bride d'admission, 3 , p. 174

Budgétisation, 4, p. 222, 223.

Bulbe duodénal, 2, p. 106.

Bulletin d'expédition, 2, p. 112.

Bus, 1, p. 65 .

BV, 1, p. 42 .

CA, 1, p. 42 .

Cachet social, 2, p. 112.

Cadres, 1, p. 39.

Calcul biliaire, 2, p. 105.

Calcul urinaire, 2, p. 105.

Calendrier de protection, 1, p. 40.

Campus, 1, p. 65.

Canalicule séminifère, 2, p. 104.

Canalisation, 2 , p. $113 ; 3$, p. 173 .

Canalisation d'incendie, 3 , p. 175

Canalisation du service journalier, 3, p. 175.

Cancer primaire du sein, 2, p. 102.

Cancer roentgenien, 2, p. 98.

Cantonnement automatique, 1, p. 42.

Caractère statique, 1, p. 28.

Carboduc, 2, p. 113

Carpien, 2, p. 104

Cataracte thermogène, 2, p. 98 .

Catarrhal, 2, p. 104.

Causatif, 1, p. 4

CCC, 1, p. 42 .

Cédétiste, 1, p. 65

Cellule falciforme, 2, p. 98 .

Cellules séminales, 2, p. 104.

Centre de la francophonie, 1, p. 53.

Cervical, 2, p. 104.

Chantier de transbordement, 2, p. 115.

Chantier multitechnique, 2, p. 115.

Chantier rail-route, 2, p. 115.

Chapeau, 3, p. 174.

Chauffage, 4, p. 233

Chaufferette, 4, p. 234

Chef de bureau, d'équipe, de service, 1, p. 40.

Chef d'entreprise, 1, p. 39.

Cher ami, 2, p. 90.

Chute de tension, 1, p. 36.

Cicatrisation, 2, p. 97.
C.I.M., 2, p. 108.

Cinéastes, 3, p. 184.

Cinétisation des prépositions, 2, p. 97.

Circulation portale, 2, p. 104.

Clapet, 3, p. 173.

Classeur, 3, p. 182

Clignotant, 4, p. 232.

Clignoteur, 4 , p. 232.

Coagulation, 2 , p. 97 .

Code orthographique, 1, p. 48 .

Colique, 2, p. 105.

Colis de service, 1, p. 42.

Colonne, 3 , p. 174.

Colonne de direction, 4, p. 233

Comité de réclamation, 3, p. 165.

Commande centralisée de la circulation, 1 , p. 42.

Commande de chauffage, 4, p. 234.

Commande du démarreur, 4 , p. 232

Commerce, 4, p. 240.

Commercialisation, 2, p. 94.

Communication, 1 , p. 31,45 .

Commutateur d'allumage, 4, p. 232.

Commutateur d'éclairage, 4, p. 232.

Commutateur de code, 4, p. 232.

Compétence, 3 , p. 150.

Compléter, 1, p. 38 .

Complication tardive, 2, p. 102.

Comptes sociaux, 2 , p. 112 .

Compteur d'incendie, 3, p. 175

Compteur de milles, 4, p. 233 .

Compteur journalier, 4, p. 233.

Compteur kilométrique, 4, p. 233.

Compteur milliaire, 4, p. 233.

Compteur totalisateur, 4, p. 233.

Concentration inhibitrice minimale, 2, p. 108.

Concret, 4, p. 203.

Concret-abstrait, 1, p. 5.

Concrétisation, 1, p. 38 .

Conduit, 2, p. 173 .

Conduite, 3, p. 173.

Conduites alimentaires, 3, p. 174.

Conduite d'eau de la ville, 3, p. 175 .

Conduite de distribution, 3, p. 174.

Conduites en impasse, 3, p. 174.

Connaissement, 2, p. 112.

Conscience linguistique, 1, p. 46.

Contact, 4, p. 232.

Contact démarreur, 4, p. 232.

Container à PA, 1, p. 42.

Contenu, 3, p. 147.

Contexte non synonymique, 3 , p. 151.

Contextes non synonymiques (exemples), 3 , p. 155.

Contexte synonymique, 3 , p. 151.

Contextes synonymiques (exemples), 3, p. 154.

Contraintes grammaticales, 1, p. 46.

Contrat de transport, 2, p. 112.

Contremaître, 1 , p. 40.

Contresens, 2, p. 89.

Contrôle, 1, p. $65 ; 3$, p. 182.

Contrôle direct, 1, p. 47.

Convenances, 1 , p. 46

Coronaire, 2, p. 104.

Coronarien, 2, p. 104

Corps riziforme, 2, p. 98 .

Corps tubulaire, 3, p. 174.

Corrections typographiques, 1, p. 48 . 
Correspondance commerciale, 1, p. 48.

Costal, 2, p. 104.

"Coton absorbant ", 2, p. 117.

Coude à patin, 3 , p. 174 .

Coulisse, 2, p. 106.

Coup de bélier, 3, p. 174.

Coupure franche, 4, p. 221.

Courbe d'option, 4, p. 223.

«Courir un cheval », 1, p. 38.

Coût d'option, 4, p. 223.

Crème de fromage, 3 , p. 176.

Crème de fromage fondu, 3, p. 178

Crête, 2, p. 106.

Crêtes palatines, 2, p. 106.

Croisement linguistique, 1, p. 31 .

CS, 1, p. 42

Cul-de-sac, 2, p. 106.

Culture, 2, p. 99

Cure de piqûres, 2, p. 103.

Danse, 3, p. 184.

Darsonvalisation, 2, p. 107

"Date d'expiration \%, 2, p. 117.

DE, 2, p. 108

Débit nécessaire à la lutte contre le feu, 3, p. 174.

Débit nominal, 3, p. 175.

Décéder, 3, p. 150 .

Déclaration d'expédition, 2, p. 112.

Dégâts, 2, p. 99 .

Dégivrage, 4, p. 233.

Dégoût, 2, p. 90

Dégroupage, 2, p. 113.

Délégation de pouvoir, 1, p. 39.

Démangeaison, 2, p. 99

"Démangeaison hivernale », 2, p. 99.

Demi-raccord, 3, p. 174.

"Dénomination », 1, p. 38

Dentaire, 2, p. 104.

Déodorant, 1, p. 65.

Déplacement de sens, 1, p. 36 .

Dépolluer, 1, p. 65.

Déposer plainte, 1, p. 65.

Dérivation adjectivale, 2, p. 99.

Dermatite cercarienne, 2, p. 97.

Désembuage, 4, p. 233.

Désembueur, 4, p. 234.

Désinences, 2, p. 104.

Desserte urbaine, 2, p. 113.

Dévalorisation sémantique, 1, p. 38.

Diagnostic précoce, 2, p. 102.

Différence de potentiel, 1, p. 35 .

Différenciation des langues, 1, p. 30.

Difficultueux, 1, p. 65.

Directeur, 1, p. 39.

Direction, 1, p. $39 ; 2$, p. 111.

"Direction », 2, p. 117.

Directive générale, 2, p. 113.

Dirigeant, 1, p. 39

Disparaître, 2, p. 91.

Dispositif d'homme mort, 1, p. 42.

Dispositif de VA, 1 , p. 42.

Dispositif de vidange, 3, p. 174 .

Distribution, 2, p. 122

Distribution au hasard, 2, p. 96

DL, 2, p. 108 .

Doctorat, 3, p. 167, 168.

Doctorat professionnel, 3, p. 170.

Documentation, 4 , p. 241 .
Dommage, 2, p. 99.

Dosage, 2, p. 96

Dose de ravnel, 2, p. 102.

Douleur à la pression, 2, p. 103

Douleur térébrante, 2 , p. 98

Douloureux à la pression, 2, p. 103.

Drain, 3, p. 174

Drame, 2, p. 92.

Droit, 4, p. 240

E., 4, p. 233.

E.C.G., 2, p. 108.

Echancrure, 2, p. 106

Economie, 4, p. 240.

Ecoulement nasal, 2, p. 103.

Ecrémeuse, 1, p. 37.

Education/instruction, 3, p. 153.

E.E.G., 2, p. 108.

Effet de style, 1, p. 47.

Eglise, 1, p. 38 .

Electrochoc, 2, p. 108.

Ellipse, 2, p. 97.

Ellipse des articles en anglais, 2, p. 100.

Eloigné, 2, p. 102

Embol, 2, p. 100.

Embolie graisseuse, 2, p. 98

Embryonnaire, 2, p. 105.

Eminence, 2, p. 106

Eminence sublinguale, 2, p. 106.

Emprunt, 2, p. 110.

Enchaîné, 4, p. 220

Endothéliome kupfférien, 2, p. 107.

Enrichisseur, 4, p. 232.

Enseignement supérieur, 4, p. 224.

En-têtes de brevets, 1, p. 52

Entrer (en parlant du vent), 2, p. 91.

Entrer dans la foule, 2, p. 92.

Envergure, 4, p. 236.

Envoi de détail, 1, p. $42 ; 2$, p. 113.

Epidémie, 2, p. 105.

Epidémique, 2, p. 105.

Epigraphe, 1, p. 65.

Epilepsie larvée, 2, p. 104

Epine, 2, p. 106.

Epithélioma baso-cellulaire, 2, p. 98.

Epithélium pavimenteux, 2, p. 98.

Epopée de Gilgamesh, 1, p. 33.

Equipement, 4, p. 240.

Equivalence stylistique, 2, p. 89.

Erythème calorique, 2, p. 98 .

Escale, 2, p. 114

"Escompte ", 1, p. 37.

Espace, 2, p. 106.

Esprits éclairés, 1, p. 29.

Essai en double anonymat avec permutations croisées, 2, p. 96.

Essais hydrauliques, 3, p. 174.

Essence de gelée, 1, p. 38 .

Essuie-phares, 4, p. 233.

Est-ce qu'on s'occupe de vous ?, 2, p. 91.

Estimation, 1, p. 41.

Etape, 2, p. 114.

Etape autorisée, 2, p. 114.

Etat mental, 2, p. 104.

Etc., 1, p. 65

Eteindre (s'), 3, p. 150.

Etrangleur, 4, p. 232.

* Etre familier avec quelque chose », 1, p. 39.

« Etre gradué », 1, p. 33. 
Etymologie, 4, p. 239.

Evaluation, 1, p. 41

Evolution (d'une maladie), 2, p. 103.

Examen du squelette, 2, p. 100.

Exceptionnel, 4, p. 233.

Exergue, 1, p. 65

Explicitation, 4, p. 210.

Explicitation des objectifs, 4, p. 222.

Exposition, 4, p. 237.

Expressionnisme, 1, p. 46.

Extension de sens, 1, p. 38

Extincteur automatique, 3, p. 175.

Extrait d'un passage contre l'adultère, 1, p. 8.

Extrait du discours de la Sagesse, 1, p. 7.

Extrant, 4, p. 223.

Faire partir, 1, p. 38.

Faire tache d'huile, 2, p. 92.

Faire une culture, 2, p. 100.

Faisceau code, 4 , p. 232.

Faisceau de croisement, 4, p. 232

Faisceau route, 4, p. 232.

Fait d'expression, 1, p. 47.

Fait stylistique, 1, p. 45.

Faute impardonnable, 2, p. 92.

Fauteuil, 1, p. 36.

Fente, 2, p. 106.

Ferme-porte, 3, p. 182.

Ferment lipolytique, 2, p. 98.

Ferret, 2, p. 112.

Feuille de route, 2, p. 112

Feux, 4, p. 232.

Feux de croisement, 4, p. 232.

Feux de route, 4 , p. 232.

Fièvre bilicuse, 2, p. 105.

Fièvre de Malte, 2, p. 105.

Fièvre épidémique d'Assam, 2, p. 105.

Fièvre folle, 2, p. 105.

Fièvre méditerranéenne, 2, p. 105.

Fièvre ondulante, 2, p. 105.

Fièvre rhumatismale, 2, p. 106.

Fièvre sudorale, 2, p. 105.

«Filière ", 3, p. 182

Films, 3, p. 184.

Fistule biliaire, 2, p. 105.

Flore 2, p. 121.

Fondu, 4, p. 220

Fondu au blanc, 4, p. 220.

Fondu au gris, 4, p. 220.

Fondu au noir, 4, p. 220

Fondu enchaîné, 4, p. 220.

Fontainerie, 3, p. 174 .

Formation, 1, p. 40

Formule brute, 2, p. 95.

Formule développée, 2, p. 95.

Formule leucocytaire, 2, p. 109.

Fossette gassérienne, 2, p. 107.

Francophonie, 1, p. 53.

Fromage à tartiner, 3, p. 177.

Fromage fondu, 3, p. 176 .

Fromage fondu pour tartine, 3, p. 176

Fromage reiait, 3, p. 177.

F.S.H., 2, p. 108 .

Futur, 1, p. 4

Gabarit passe-partout, 2, p. 114

Gaine, 3, p. 173.

Garantie, 1, p. 38 .

Gazoduc, 2, p. 113.

“ Gérant », 1, p. 39.
Germinatif, 2, p. 104.

Gestion, 1, p. 39; 2, p. 110.

Gestionnaire, 2, p. 111.

Glande sudoripare, 2, p. 98 .

Glycémie, 2, p. 97.

Gogo, 1, p. 37

"Goûter" ", 1, p. 38.

Goutte tophacée, 2, p. 98.

Gouttière, 2, p. 106.

Grade avancé, 4, p. 225, 230.

Grades universitaires, 3, p. 166; 4, p. 224

Gradin, 1, p. 37 .

"Gradué », 1, p. 39.

Grammaire, 1, p. 7, 47

Grammairiens et linguistes, 1, p. 62.

Grammatical/idiomatique, 2, p. 91.

Grands services, 2, p. 112.

Granoduc, 2, p. 113 .

Granules pigmentaires, 2, p. 98 .

Grief, 3, p. 164.

Grille-pain, 3, p. 182.

"Gros flacon », 2, p. 96.

Grossesse gémellaire, 2, p. 98 .

Grossesse tubaire, 2, p. 107.

Groupage, 2, p. 113.

Groupe mobile, 3, p. 182.

Groupeur, 2, p. 113.

Hagiographie, 4, p. 240.

Happening, 1, p. 65.

Hébreu, 1, p. 3.

Hémoglobinophathie à hématies falciformes, 2, p. 100.

Hémorragie intestinale massive, 2, p. 9.

Hémorroïdal, 2, p. 104.

Hespéranopie, 2, p. 97.

Homme mort, 1, p. 42.

Hydrothérapie, 2, p. 97.

Hydrochrome, 2, p. 105.

I.C.S.H., 2, p. 108 .

Idée d'université, 1, p. 26

Idiomatique/grammatical, 2, p. 91.

Ignorance des idiomes, 1, p. 63.

Ilot, 2, p. 92.

I.M., 2, p. 108

Image directe, 2, p. 92.

Image lacunaire, 2, p. 99.

Imparfait, 1, p. 4

Impérialisme de la technique, 1, p. 60.

Implicat de diversité, 3 , p. 152.

Importance de la traduction, 2, p. 129

Impressionnisme, 1, p. 46.

Inconciliable, 1, p. 37 .

Incongelable, 3, p. 174

Index du sélecteur, 4, p. 233.

Indicateur de vitesse, 4, p. 233.

Infection hydrique, 2, p. 98 .

Influence de la traduction sur la langue, 2, p. 129.

Informatique, 3, p. 185.

Ingénierie, 3, p. 182.

"Initial ", 2, p. 102.

Instruction/éducation, 3, p. 153.

Intensif, 1, p. 4.

Intensité, 1, p. 36

Interprétation, 1, p. 32.

Intertrochantérique, 2, p. 105.

Intransitivation, 1, p. 38 .

Intrant, 4, p. 223. 
Inverseur de courant, 4, p. 232. Inversion, 1, p. 39.

« Irréconciliable », 1, p. 37.

I.V., 2, p. 108

Jointures, 1, p. 28.

Joli, 3, p. 150.

Journalisme, 2, p. 121.

Juke-box, 1, p. 65.

Jussif, 1, p. 4.

Kala-azar infantile, 2, p. 105.

Kérosène, 1, p. 65.

Kilomètre, 2, p. 94

Kitchennette, 1, p. 65.

Klaxon, 4, p. 232.

"Label $», 2$, p. 117.

Lacune, 2, p. 99.

Lacune du champ visuel, 2, p. 99.

Laisse, 1, p. 18.

Lances d'incendie, 3, p. 174.

Langage médical, 2, p. 127.

Langue, 2, p. 76 .

Langues/langage, 1, p. 31.

Langue administrative, 3 , p. 185.

Langue artificielle, 3, p. 159.

Langue dépapillée, 2, p. 98 .

Langue des affaires, 1, p. 48.

Langue française, 2 , p. 137.

Langue française et expression scientifique, 1, p. 62.

Langue historique, 3, p. 159.

Langue inhumaine, 3 , p. 159

Langue naturelle, 3, p. 153.

Langue objective, 3 , p. 153 .

Langue pure, 1, p. 31 .

Langue sémitique, 1, p. 3.

Langue tahitienne, 3 , p. 184 .

Langue universelle, 1, p. 30.

Larvé, 2, p. 104.

Lave-phares, 4 , p. 232.

Léser, 2, p. 99.

Lésion, 2, p. 99.

Lettre de transport aérien, 2, p. 112

Lettre de transport ferroviaire, $2, \mathrm{p}$. 112.

Lettre de transport maritime, 2, p. 112.

Lettre de transport routier, 2 , p. 112.

Lettre de voiture, 2, p. 112.

Levier au plancher, 4, p. 233.

Levier de changement de vitesse, 4, p. 233

Levier de dégagement, 4, p. 233.

Levier de vitesse, 4, p. 233.

Levier sélecteur de vitesse, 4, p. 233.

Lexicographie, 1, p. 7; 2, p. 140.

Licence, 3 , p. 167.

Ligament, 2 , p. 106

Ligament tendinotrochantérien, 2, p. 105.

Ligne, 2, p. 106.

Ligne intertrochantérique, 2, p. 105.

Limbique, 2, p. 105

Linguistique, 1, p. 55, 56.

Linguistique allemande, 2 , p. $122 ; 3$, p. 186.

Linguistique anglaise, 2, p. 122; 3, p. 186.

Linguistique arabe, 2 , p. 122; 3, p. 186.

Linguistique asiatique, 3, p. 186 .

Linguistique différentielle, 2, p. 92.

Linguistique espagnole, 2, p. 122.

Linguistique générale, 2, p. 124;3, p. 187;4, p. 242.
Linguistique française, 2 , p. $122 ; 3$, p. $187 ; 4$, p. 242.

Linguistique japonaise, 2 , p. 122

Linguistique slave, 2 , p. 122.

Liséré, 2, p. 106.

Lishmaniose splénique infantile, 2, p. 105.

Littéralité, 2, p. 88.

Livre des proverbes, 1, p. 3 .

L.H., 2, p. 108.

Logement, 1, p. 36

Logeur, 2, p. 90.

Long rail soudé, 1, p. 42.

Lordose, 2, p. 97

LRS, 1, p. 42.

Luxation acomio-claviculaire, 2, p. 98

Lymphadénie splénique des nourrissons, 2, p. 105 .

Lymphogranulomatose maligne, 2, p. 105.

Macrodactylie, 2, p. 97.

Maillé, 3, p. 174.

Main, 3, p. 173.

Main phocomélique, 2, p. 98

Maîtrise, 1, p. 40; 3, p. 167,$170 ; 4$, p. 230.

Maladie d'Apert, 2, p. 107.

Maladie de Banti, 2, p. 107.

Maladie de Basedow, 2, p. 107.

Maladie de Bouilland, 2, p. 106

Maladie de Graves, 2, p. 107.

Maladie de Hein-Médin, 2, p. 105.

Maladie de Marsh, 2, p. 107.

Maladie de Paget, 2, p. 107.

Maladie de Recklinghausen, 2, p. 107

Maladie osseuse de Paget, 2, p. 107.

Maladie sérique, 2, p. 98.

Malformation, 2, p. 99.

Malthusianisme lexical, 1, p. 62.

"Management ", 2, p. 110.

Manche à incendie, 3 , p. 173 .

Manches souples, 3, p. 173.

Mandarinat du purisme, 1, p. 61.

Maniaque, 2, p. 105.

"Manquer quelqu'un », 1, p. 39.

Marche à suivre, 1, p. 40 .

Marche arrière, 4, p. 233.

Marcher comme sur des roulettes, 2, p. 92.

Marge, 2, p. 106.

Marteau d'eau, 3, p. 174.

Massétérin, 2, p. 105.

Matraquage, 1, p. 65.

Mauvaise vue, 2, p. 99.

Maxillaire, 2, p. 105.

Méfaits de la traduction, 2, p. 132

Mélitococcie, 2, p. 105.

Mentonnière, 2, p. 104.

Métalangue, 3, p. 153.

Métaphore, 2, p. 92.

Méthodes, 1, p. 40.

Mettre en marche, 1, p. 38

Micheline, 2, p. 113.

Millage, 3, p. 182.

Mille, 2, p. 94.

Milliaire, 4, p. 233.

Minuterie d'extinction des phares, 4, p. 232.

Mode opératoire, 1 , p. $40 ; 2$, p. 96 .

Modernisation de vocabulaire bilingue, 1, p. 6 .

Monème, 3, p. 151.

Mcrphème, 3, p. 151.

Mot, 3, p. 147 . 
Mot image, 2, p. 92.

Mot signe, 2, p. 92.

Moteur, 3, p. 175 .

Moteur à vapeur, 3, p. 175 .

Moteur diésel, 3, p. 175.

Moteur électrique, 3 , p. 175 .

Moto-pompe fixe, 3, p. 175.

« Moulin à papier », 3, p. 182.

Mourir, 3, p. 150.

Mouvement de la phrase, 1, p. 47.

Mouvement littéraire, 1, p. 47.

Muscle: du squelette, 2, p. 100.

Musiciens, 3, p. 184.

Musique, 3, p. $184 ; 4$, p. 240.

Musique de la phrase, 1, p. 47 .

Myopie, 2, p. 97.

Myorelâchant, 2, p. 96.

Mythe de la pureté linguistique, 1, p. 60 .

$\mathrm{N}, 4$, p. 233.

Néerlandais, 4, p. 203.

Néerlandophone, 4, p. 204.

Neurofibromatose, 2, p. 107.

Neutre, 4, p. 233.

Niphablepsie, 2, p. 97.

Niveau de langue, 1, p. 49, 63.

Noms communs, 3, p. 147.

Norme et usage, 1, p. 60.

Norme immanente, 1, p. 61

Notes (rédaction des), 1, p. 48

Note circulante, 1, p. 42

Nuances qualitatives, 1, p. 46

Nuances quantitatives, 1, p. 46

Nuitée, 1, p. 65

Objet, 3, p. 147.

Objectif, 1, p. 40; 4, p. 222.

Obtenir son diplồme, 1, p. 39.

Obtenir un grade, 1, p. 39.

Odomètre, 4, p. 233.

Ecuménisme, 1, p. 3.

CEuvre littéraire, 1, p. 45.

Oléoduc, 2, p. 113.

Onde coronarienne, 2, p. 105.

Opération de Finsterer, 2, p. 107.

Opération de Hofmeister, 2, p. 107.

Opération de Péan, 2, p. 107.

Opération de Reichel, 2, p. 107.

Ophtalmie des neiges, 2 , p. 97.

Option, 4, p. 223.

Ordonnance prosodique, 1, p. 19.

Ordre des mots, 1, p. 47.

Organisation, 1 , p. $39 ; 2$, p. 110

Orifice d'écoulement, 3, p. 174.

Ostéite déformante, 2, p. 107.

Ostéite fibrokistique, 2, p. 107.

Ourlet, 2, p. 106.

P, 4, p. 233.

PA, 1, p. 42

Palatine, 2, p. 104.

Pansement occlusif, 2, p. 100.

Papeterie, 3, p. 182.

"Papier de toilette », 2, p. 117.

Papilles gustatives, 2, p. 98 .

Paralysie spinale infantile, 2 , p. 105.

Parfait, 1, p. 4.

Parfum (de glace), 1, p. 38.

Parking, 4, p. 233.

Parler, 3, p. 162

Parlers régionaux, 1, p. 60.
Parole, 3, p. 151.

Particularisation, 1, p. 36 .

"Partir un moteur », 1, p. 38.

Passage à niveau, 1 , p. 42 .

Passé, 2, p. 102

Passivation, 1, p. 38.

P.C.E., 2, p. 108.

Péché, 2, p. 92.

Pensée, 1, p. 45

Performance, 3, p. 150.

Période mewmanienne, 1, p. 27.

Perte de charge, 1 , p. $36 ; 3$, p. 174.

Phare, 4, p. 232.

Phare-code, 4, p. 232.

Phare-route, 4, p. 232

Pharmacologie dentaire, 4, p. 240.

Phonème, 3, p. 147.

Pipe-line, 2, p. 113.

Piquer sur, 3, p. 174.

Placentaire, 2, p. 104.

Plainte d'un tramway, 2, p. 93.

Plan, 1, p. $40 ; 4$, p. 222

Plan de dépenses, 4, p. 223.

Plan de l'entendement, 4, p. 204.

Plan du réel, 4, p. 204.

Planification, 1, p. 39, 40; 4, p. 222.

Planning, 1, p. 40

Plaque de regard, 3 , p. 175.

« Plaster », 2, p. 117.

Pléthorelexicale, 1, p. 60

Pli de service, 1, p. 42.

Pli fessier, 2, p. 106

PN, 1, p. 42.

Poche, 2, p. 92.

Points-voyelles, 1, p. 3.

Poire, 1, p. 37.

"Poisson ", 1, p. 37

Pollution, 2, p. 122.

Polonais, 3, p. 184.

Polyarthrite aiguë fébrile, 2, p. 106.

Polyarthrite chronique évolutive, 2, p. 106, 108.

Polyarthrite déformante, 2 , p. 105

Polyarthrite rhumatoïde, 2, p. 106.

Polymorphie, 3, p. 159.

Polyomyélite antérieure aiguë, 2, p. 105.

Polysémie, 3, p. 148.

Pomme de pin, 2, p. 90 .

Pomperie, 3, p. 175 .

Ponctuation électronique, 4, p. 220.

Porte(veine), 2, p. 104

Porte-conteneur, 2, p. 115.

Porte coupe-feu, 3, p. 175.

Porteur aménagé (à), 1, p. 42.

Position, 2, p. 101.

Posologie, 2, p. 96.

Posologie d'entretien, 2, p. 102.

Posséder, 1, p. 37.

Poste de contrôle, 3, p. 175.

Poster, 1, p. 65.

Poteau d'incendie, 3, p. 174

Précision, 1, p. 36.

Précoce, 2, p. 102.

Précommercial, 2, p. 95.

Prédicat-sujet, 1, p. 4.

Prédicateur, 3, p. 147.

Préjudice, 2, p. 99.

Présent d'habitude, 2, p. 91. 
Prévenu, 1, p. 38.

Primaire, 2, p. 102.

Prise de refoulement, 3, p. 175.

Prise principale de face, 3, p. 174.

Prises secondaires latérales, 3 , p. 174.

Procédé, 1, p. 40.

Procédé d'analyse, 2, p. 96.

Procédé de Plya, 2, p. 107

Processus, 2, p. 106.

Processus décisionnel, 4, p. 222.

Produit grossissant, 2, p. 96.

Professions (enseignement des), 3, p. 168.

Programmation, 4, p. 222.

Projet, 4, p. 223.

Projet d'information wagons, 1, p. 42.

Prolongement, 2, p. 106.

Propriété industrielle, 1 , p. 53.

Prospective, 4, p. 222.

Prospective-plan-budget, 4, p. 222.

Proverbes, 1, p. 54.

Prurit hivernal, 2, p. 99.

PS, 1, p. 42.

Pseudoleucémie infantile, 2, p. 105.

Pseudoleucémique, 2, p. 105.

Pseudonymes, 4, p. 240.

Psychologie, 1, p. 55; 2, p. 120.

Publications scientifiques de langue française, 1, p. 62.

Publicité, 1, p. 63

Puis-je vous aider?, 2, p. 91.

Pulmonaire, 2, p. 104.

Quai, 2, p. 115.

"Q-Tips ", 2, p. 117

R.A.A., 2, p. 106.

Rabais, 1, p. 37.

Raccord pompier, 3, p. 175.

Rail-route, 2, p. 115.

Rainure, 2, p. 106.

Raison sociale, 2, p. 112.

Ramifié, 3, p. 174 .

Rampe rail-route, 2 , p. 115.

Raphé, 2, p. 106.

Rapports (rédaction des), 1, p. 48.

Rationalisation des choix budgétaires, 4, p. 222.

$\mathrm{RCB}, 4$, p. 222.

Réaction de fixation du complément, 2, p. 108.

Réalités commerciales, 1, p. 7.

Réaménagement lexical, 1, p. 61

Recherche opérationnelle, 4, p. 223.

Réclamation, 3, p. 164.

Réclamer, 3, p. 165.

Reconnaissance automatique des wagons, 1 , p. 42.

Rédaction des rapports, notes et communications, 1 , p. 48

Réduction, 1, p. 37.

Réflexe massétérin, 2, p. 98.

"Regarder », 1, p. 38 .

Régionalisme, 1, p. 60 .

Régulateur de vitesse, 4, p. 233

Relations extérieures, 2 , p. 116.

Relations publiques, 2, p. 116.

Remise, 1, p. 37.

Rénal, 2, p. 104.

Rencontre, 1, p. 31

Renverser, 2, p. 92.

Répartition des responsabilités, 1, p. 39 .
Repli, 2, p. 106.

Repli sublingual, 2, p. 106

R.E.R., 2, p. 113 .

Réseau d'adduction et de distribution, 3, p. 174.

Réseau d'eau, 3, p. 173, 174 .

Réseau d'eau d'incendie, 3, p. 175 .

Réseau d'eau industrielle, 3, p. 175.

Réseau express régional, 2. p. 113.

Responsabilité, 4, p. 236.

Ressources en eau, 3, p. 174

Résultat immédiat, 2, p. 102.

Rétinien, 2, p. 104.

Rhinorrhée, 2, p. 103.

Rhumatisme articulaire aigu, 2, p. 106

Rhumatisme chronique déformant, 2 , p. 106

Rideau, 4, p. 221.

Rideau coupe-1 eu, 3, p. 175.

Rideaü d'eau, 3, p. 175.

Rideau horizontal, 4, p. 221.

Rideau vertical, 4 , p. 221 .

Risque voisin, 3, p. 175.

Robinet, 3, p. 174 .

Robinet armé, 3, p. 175.

Rôle de traduction dans le monde, 2, p. 129.

Sacré, 2, p. 104.

Saignement, 2, p. 99.

Saignement de nez, 2, p. 99

Salivaire, 2, p. 104.

Sanctionner, 1, p. 65.

Sarcome giganto-cellulaire, 2, p. 98.

Saumoduc, 2, p. 113.

Sauver (se), 2, p. 92.

« Saveur », 1, p. 38 .

Schistosomiase cutanée, 2, p. 97.

Sciences naturelles, 2, p. 90.

Sciure simienne, 2, p. 98 .

Sculpture, 4, p. 240.

S'en laver les mains, 2, p. 92.

Secteur, 4, p. 233.

"Sécurité", 1 , p. 38

Sélecteur, 4, p. 233

Sentiment, 3, p. 150.

Séminifère, 2, p. 104.

Sens de la traduction, 1, p. 29.

"Séparateur », 1, p. 37.

Septante, 1, p. 5.

Services généraux, 2, p. 113.

"Serviette sanitaire », 2, p. 117.

Siège, 1 , p. 36

"Siéger sur un comité », 4, p. 215.

Signe, 3, p. 147.

Signes de ponctuation électronique, 4, p, 220.

Sillon, 2, p. 106.

Sillon fessier, 2, p. 106.

Sillon latéral de l'ongle, 2, p. 106.

Simple soldat, 2, p. 90.

Situation actuelle du traducteur, 2, p. 131

Situation de la langue française, 2, p. 128.

Ski, 3, p. 185.

S.L.I., 2, p. 109.

S.N.C., 2, p. 108

Social, 2, p 112.

Somptuaire, 1, p. 65.

Sortie d'hôpital, 2, p. 103.

Souillard, 3, p. 175

Soupape, 3, p. 173.

Sous-programme, 4, p. 223. 
Sous-sous-programmes, 4, p. 223.

Sous-unguéal, 2, p. 104.

Spasme acétylcholonique, 2 , p. 96.

Spécialisation, 3, p. 172.

Squelette, 2, p. 99

"Squelettique $\approx, 2$, p. 100

Substitution catégorielle, 2, p. 97,99

* Suce *, 2, p. 117.

Suites éloignées de la transplantation, 2, p. 102.

Supermarché, 2, p. 122.

Supervision, 1, p. 41.

Sur/on, 4, p. 212.

Sur la rue, 4. p. 214.

Sur le train, 4, p. 214

Surintensité, 1, p. 36.

Surplomber, 1, p. 65.

Surpresseur, 3, p. 175

Surtension, 1, p. 36.

Surveillance immédiate, 1, p. 41.

Surveillant, 1, p. 40.

Stage, 1, p. 40.

Stance sémitique, 1, p. 18.

« Starter ", 4, p. 232.

Stiche, 1, p. 18

Stique, 1, p. 18

Stratégie, 4, p. 222.

Stratigraphie, 2, p. 106

Streptococcique, 2, p. 104

Strie, 2, p. 106

Strophe psalmique, 1, p. 18.

Strophe sémitique, 1, p. 18.

Strc phique des psaumes, 1, p. 18.

Structure, 2, p. 89

Structure/pensée, 2, p. 89.

Structure de l'entreprise, 1, p. 40

Style, 1, p. 45, 47; 4, p. 230.

Style biblique, 1, p. 3.

Style commercial, 1, p. 50.

Style courant, 4, p. 236.

Style d'information, 4 , p. 237.

Stvle familier, 4, p. 237.

Style en fonction de la postérité, 4, p. 238.

Style intime, 4, p. 237.

Style nominal, 4, p. 205.

Stylistique, 1, p. 46, 47.

Stylistique comparée, 2, p. 89; 4, p. 203.

Symboles, 2. p. 120.

Syndrome d'Adair-Dighton, 2, p. 107

Syndrome de Lobstein-Cornazon, 2, p. 107.

Synorlymie, 3, p. 147, 148.

Synoniymiaue, 3 , p. 149

Synonymité, 3 , p. 150.

Syntagmex nominal, 4 , p. 205.

Système multiclignotant, 4, p. 232

Système verbal de l'hébreu, 1, p. 4 .

Tabernacle, 3 , p. 174 .

Tableau de bord, 4, p. 231

Tactique, 4, p. 222.

Tardif, 2, p. 102.

Tartan, 1, p. 65.

Taux d'actualisation, 4, p. 222

Taux de glycémie, 2, p. 101.

Technique dite UFR, 2, p. 114.

Technique kangourlu, 2, p. 114

Technique MC 22, 2, p. 114

Technologie laitière, 3 , p. 178

Temporal, 2, p. 104
Temps, 1, p. 4 .

Tendinotrochantérien, 2 , p. 105 .

Tension, 1, p. 35 .

Tension d'exploitation, 1, p. 36 .

Termes de brevet, 1, p. 52 .

Termes de médecine, 2, p. 122.

Termes modernes, 1, p. 6

Termes pharmaceutiques, 2, p. 117

Termes routiers, 1, p. 5 .

Terminologie, 2 , p. 140

Terminologie judiciaire, 2, p. 121.

Terminologie juridique. 3 , p. 185.

Terminologue, 1, p. 52.

Tétanos, 2, p. 97.

Texte Massorétique, 1, p. 4.

Thématiser, 3, p. 151.

Thésaurismose, 2, p. 97.

Thèse, 3, p. 169 .

Tic de nutation, 2, p. 98 .

Tige de commande, 3 , p. 174.

Tirette, 4, p. 232

Tireuse optique, 4, p. 221.

Tob, 1, p. 4.

Tomographie, 2, p. 106.

Tophus, 2, p. 97.

Tort, 2, p. 99

Tour de Babel, 1, p. 31

Traduction, 1, p. 32; 3, p. 186 .

Traduction scientifique française, 1, p. 62 .

Traduction au Canada, 2, p. 130.

Traduction automatique, 4, p. 242.

Traduction de contact, 3, p. 178.

Traduction de néerlandais, 4, p. 203.

Traduction de langues des affaires, 1, p. 48.

Traduction et le français, 2 , p. 133 .

Traduction exacte, 2, p. 94

Traduction humaine, 4, p. 242.

Traduction (journée de la), 3 , p. 191

Traduction libre, 2, p. 88

Traduction littérale, 1, p. $5 ; 2$, p. 88

Traduction médicale, 2, p. 95 .

Traduction médico-pharmaceutique, 2, p. 95.

Traduction cecuménique de la Bible, 1, p. 4.

Traduction religieuse, 1, p. 1 à 34 .

Tragédie, 2, p. 92.

Trafíc de détail, 1, p. 42.

Trafic de groupage, 2, p. 113.

Transitivation, $1, \mathrm{p}, 38$.

Transplantation, 2, p. 102

Transport combiné, 2, p. 115 .

Transport de détail, 1, p. 42.

Transporteur, 2, p. 113.

Travail d'équipe, 1, p. 5

Travailler, 4. p. 219.

Travaux publics, 4, p. 240

Trochantérien, 2, p. 105.

Trochantériaue, 1, p. 105.

Trou de mémoire, 1, p. 38.

Truander, 1, p. 65 .

Truca, 4, p. 221.

Truqueur électronique, 4 , p. 221

Tubercule, 2, p. 106.

Turbine à vapeur, 3, p. 175

Tuyau rigide, 3 , p. 173 .

Tuyau semi-rigide, 3, p. 173.

Tuyautage, 3 , p. 173

Tuyauterie, 3 , p. 173

Tympanique, 2, p. 105. 
U.I.V., 2, p. 108.

Unité de traduction, 2, p. 90 .

Unité élémentaire de structure, 4, p. 223.

*Unité mobile ", 3, p. 182

Unité organisationnelle, 1, p. 40.

Universalité de la norme, 1, p. 60.

Urgence, 2, p. 103.

Urgent, 2, p. 103.

Urineux, 2, p. 104.

Urographie intraveineuse, 2, p. 109.

VA, 1, p. 42.

Vague, 2, p. 104.

Vaisseau coronaire, 2, p. 105.

Vallées ciliaires, 2, p. 106.

"Valoir », 1, p. 37.

Valve, 3, p. 173.

Valvule inférieure du canal nasal, 2, p. 106.

Vanne, 3, p. 174.

Vanne commandée par clef à béquille, 3 , p. 174.

V.B.P., 2, p. 108.

Veille automatique, 1, p. 42 .

Veine porte, 2, p. 104.

VEM, 2, p. 109.

VEMS, 2, p. 109

Ventilateurs, 3, p. 173.

Verbes français, 2, p. 119

Vers francais, 2, p. 118.

Version autorisée, 2, p. 93.

Vétéro-testamentaire, 1, p. 4, 18.
«Vials », 2, p. 117.

Vice, 2, p. 99 .

Vice de la parole, 2, p. 99.

Ville-route, 4, p. 233.

Virtualités du francais, 1, p. 61 .

Vocabulaire, 1, p. 47.

Vocabulaire commun, 1, p. 5 .

Vocabulaire médical, 4, p. 241.

Vocabulaire newmanien, 1 , p. 28

Vocabulaire technique, 3, p. 185.

Voie d'abord, 2, p. 102.

Voile, 3, p. 185.

Volant inclinable, 4, p. 233.

Volet, 4, p. 221.

Volet à compas, 4, p. 221.

Volet à battant, 4, p. 221.

Volet-cahier, 4, p. 221.

Volet circulaire, 4, p. 221.

Volet de départ, 4, p. 232.

Volet progressif, 4, p. 221.

Volt, 1, p. 35

"Voltage ", 1, p. 35.

Vous désirez?, 2, p. 91.

VR, 4, p. 233.

Vue directe, 2, p. 92.

Wagon de groupage, 2, p. 113.

Xérodermie, 2, p. 97.

Zone d'option, 4, p. 233.

Zone réflexogène, 2, p. 98. 